\title{
Article
}

\section{Synchronous and a-synchronous machines (SAASMs): rotor and stator faults}

\author{
Babar Azeem ${ }^{1, *}$, Zahid Ullah ${ }^{1}$, Faisal Rehman ${ }^{2}$, Sahibzada Muhammad Ali ${ }^{3}$, Bilal Arif ${ }^{1}$ and Aun Haider ${ }^{1}$ \\ 1 Department of Electrical Engineering, University of Management and Technology Lahore, Sialkot Campus, \\ Pakistan.; babar.azeem@skt.umt.edu.pk; (B.A) zahid.ullah@skt.umt.edu.pk (Z. U), bilal.arif@skt.umt.edu.pk (B. A) \\ aun.haider@skt.umt.edu.pk (A.H) \\ 2 Department of Electrical Engineering, Cheanab College of Engineering \& Technology, Gujranawala, Pakistan.; \\ faisal.ccet@gmail.com \\ 3 Department of Electrical Engineering, COMSATS University Islamabad, Abbottabad Campus, Pakistan.; \\ hallianali@ciit.net.pk \\ * Correspondence: babar.azeem@skt.umt.edu.pk; Tel.: +923325885944
}

Received: 11 March 2019; Accepted: 25 March 2019; Published: 23 April 2019.

\begin{abstract}
This paper investigates the rotor and stator faults of synchronous and asynchronous machine. We studied major and minor faults and failures in synchronous and asynchronous machines (SAASMs) to avoid excessive downtime, maintaining quality of service, and minimum revenue losses to smart grid (SG) operators and planners. Further, faults detection, faults diagnosis, major causes of faults, and fault remedial measures are discussed with state-of-the-art work for: (a) transformers, (b) stator, and (c) rotor. Our work presents detailed taxonomy of rotor and stator faults, electrical and mechanical stress, and faults diagnosis schemes for stable SG operation. We believe that our research contribution is more versatile covering every aspect of SAASMs faults and failures, compared to prior works.
\end{abstract}

Keywords: Transformer, stator and rotor, synchronous machines, asynchronous machines.

\section{Introduction}

$\mathbf{T}$ hree phase SAASMs are workhorse of all industries due to its versatility and robustness. However, such machines cause limitations on exceeding that cause premature failure of the stator, rotor, and shaft. Main short-comings in SAAMs includes: (a) stator winding fault failures, (b) shaft eccentricity failure, and (c) rotor faults. Damages in SAASMs are due to following reasons:

- Due to defective bearings, rotor starts vibration and results in damage of machine.

- Turns of stator winding are insulated from each other by di-electric medium, when insulation failure or short circuit occurs and machine stops working. This type of fault results in crack or damage to rotor bars where it touches end rings [1,2].

Numerous literature reports and reviews exist on SAASMs, for example Zhang et al. discussed fault detection and diagnosis system that can detect different type of faults in [3]. Stator current and time are used as inputs to system and direct torque control technology (DTC) is used as technical control in drive system. Time is factor that plays an important role for both detection of fault and selection of most appropriate corrective action in accordance with type of fault [4]. Benefits of DTC include: (a) relatively simple construction, and (b) very good flow and torque control performance. There is no transformation in power modulation block and no current control loops. Although discussed technology has some drawbacks such as: (a) variable switching frequency, (b) operating at low speed, and (c) high torque ripples. Vijayakumar et al. presented simulation of most common faults in three-phase squirrel cage machines with finite element method in [5]. Finite elements software is used to graphically record: (a) electric and magnetic fields, and (b) waveform of flux density distribution in air gap and electromagnet core. Analysis and data processing of aforementioned software is done using MATLAB. Moreover, broken rotor winding and inter-turn short circuit stator faults are studied in [6]. Results of this study show that broken rotor bars and inter-turn short circuit affects flux density in air gap. Table 1 illustrates the various stresses of the machine with respect to stator. 
Table 1. Various stresses effects on SAASMs

\begin{tabular}{|c|c|c|}
\hline & Stator & Rotor \\
\hline Electrical Stresses & $\begin{array}{l}\text { - Dielectric aging } \\
\text { - Corona } \\
\text { - Transients } \\
\text { - Tracking }\end{array}$ & $\begin{array}{l}\text { - Shear } \\
\text { - Loose lamination } \\
\text { - Thermal overload }\end{array}$ \\
\hline Mechanical Stresses & $\begin{array}{l}\text { - Rotor strikes } \\
\text { - Flying objects }\end{array}$ & $\begin{array}{l}\text { - Casting vibration } \\
\text { - Loose lamination } \\
\text { - Incorrect shaft \core fit } \\
\text { - Geometry } \\
\text { - Material deviations } \\
\text { - Part breakage }\end{array}$ \\
\hline Thermal Stresses & $\begin{array}{l}\text { - Thermal aging } \\
\text { - Voltage variation } \\
\text { - No proper Ventilation }\end{array}$ & $\begin{array}{l}\text { - Thermal overload } \\
\text { - Thermal unbalance } \\
\text { - Excessive rotor losses } \\
\text { - Sparking }\end{array}$ \\
\hline Environmental Stresses & $\begin{array}{l}\text { - Moisture } \\
\text { - Chemical } \\
\text { - Damaged parts }\end{array}$ & $\begin{array}{l}\text { - Contamination } \\
\text { - Temperature }\end{array}$ \\
\hline Dynamic Stresses & & $\begin{array}{l}\text { - Vibration } \\
\text { - Rotor rub } \\
\text { - Centrifugal force } \\
\text { - Over speeding }\end{array}$ \\
\hline
\end{tabular}

The abbreviation used in this paper are explained in Table 2. Important parameters that were discussed in the selected research papers are listed below in the comparative tabular analysis. In Table 3, ' $\mathcal{J}^{\prime}$ justifies the presence of feature while ' $\boldsymbol{X}$ ' justifies that feature is not discussed in the referred study.

Table 2. Abbreviations

\begin{tabular}{||cccc||}
\hline Abbreviation & Full-form & Abbreviation & Full-form \\
\hline \hline BB & Broken Bars & BF & Bearing Faults \\
DE & Dynamic Eccentricity & SCS & Stator Current Sensor \\
SF & Shaft Faults & RCS & Rotor Current Sensor \\
BR & Broken Rings & SST & Stator shorted Turns \\
VCS & Vibration and Current Sensors & ECS & Eccentricity Current Sensor \\
SFM & Stray Flux Measurements & EVS & Eccentricity Vibration Sensor \\
ACS & Air Coil Sensor & BRB & Broken Rotor Bars \\
BVS & Bearing vibration sensor & BCS & Bearing Current Sensor \\
\hline
\end{tabular}

\section{SAASMs rotor faults}

Three phase IMs are extensively employed in industrial and domestic applications and is helpful for practical implementation of SG due to its efficiency, durability, and robustness. Successful and safe operation of electric machines is important for maximum economy and high efficiency in industry. Monitoring of machine parameters in industry is very important to recognize and diagnose failures of electrical machine. IM performance is affected by the defects, namely: (a) electrical failures, (b) mechanical failures, and (c) environmental disturbances. Electrical failures consist of (a) asymmetric faults, (b) under voltage fault, (c) overload, and (d) fault earth leakage. Rotor winding failure, failure of stator winding and bearings 
Table 3. Summary of some generic state of art surveys

\begin{tabular}{||ccccccccccccccccc||}
\hline Papers & BB & SST & BVS & BF & BCS & ACS & BR & SCS & DE & RCS & BRB & EVS & SF & ECS & VCS & SFM \\
\hline \hline$[2]$ & $\checkmark$ & $\checkmark$ & $x$ & $\checkmark$ & $x$ & $x$ & $x$ & $\checkmark$ & $x$ & $\checkmark$ & $x$ & $\checkmark$ & $\checkmark$ & $x$ & $x$ & $\checkmark$ \\
{$[3]$} & $\checkmark$ & $x$ & $x$ & $\checkmark$ & $\checkmark$ & $x$ & $x$ & $x$ & $x$ & $\checkmark$ & $\checkmark$ & $x$ & $\checkmark$ & $\checkmark$ & $x$ & $x$ \\
{$[6]$} & $x$ & $\checkmark$ & $\checkmark$ & $x$ & $x$ & $x$ & $x$ & $\checkmark$ & $\checkmark$ & $x$ & $x$ & $\checkmark$ & $x$ & $x$ & $\checkmark$ & $x$ \\
{$[7]$} & $\checkmark$ & $x$ & $x$ & $\checkmark$ & $x$ & $x$ & $x$ & $\checkmark$ & $x$ & $\checkmark$ & $\checkmark$ & $x$ & $x$ & $\checkmark$ & $x$ & $x$ \\
{$[8,9]$} & $\checkmark$ & $\checkmark$ & $x$ & $\checkmark$ & $x$ & $x$ & $\checkmark$ & $\checkmark$ & $x$ & $\checkmark$ & $x$ & $x$ & $x$ & $x$ & $\checkmark$ & $\checkmark$ \\
{$[10]$} & $x$ & $\checkmark$ & $x$ & $\checkmark$ & $\checkmark$ & $x$ & $\checkmark$ & $\checkmark$ & $x$ & $\checkmark$ & $x$ & $x$ & $x$ & $\checkmark$ & $\checkmark$ & $\checkmark$ \\
{$[11]$} & $x$ & $\checkmark$ & $x$ & $x$ & $\checkmark$ & $x$ & $x$ & $\checkmark$ & $x$ & $\checkmark$ & $x$ & $x$ & $x$ & $\checkmark$ & $x$ & $\checkmark$ \\
{$[12]$} & $\checkmark$ & $x$ & $x$ & $x$ & $\checkmark$ & $x$ & $\checkmark$ & $\checkmark$ & $x$ & $\checkmark$ & $\checkmark$ & $\checkmark$ & $\checkmark$ & $x$ & $x$ & $x$ \\
{$[13]$} & $x$ & $x$ & $x$ & $x$ & $\checkmark$ & $x$ & $x$ & $\checkmark$ & $\checkmark$ & $\checkmark$ & $\checkmark$ & $x$ & $x$ & $\checkmark$ & $x$ & $\checkmark$ \\
{$[14]$} & $x$ & $\checkmark$ & $x$ & $x$ & $x$ & $\checkmark$ & $\checkmark$ & $\checkmark$ & $\checkmark$ & $\checkmark$ & $x$ & $x$ & $\checkmark$ & $\checkmark$ & $x$ & $x$ \\
Our Survey & $\checkmark$ & $\checkmark$ & $\checkmark$ & $\checkmark$ & $\checkmark$ & $\checkmark$ & $\checkmark$ & $\checkmark$ & $\checkmark$ & $\checkmark$ & $\checkmark$ & $\checkmark$ & $\checkmark$ & $\checkmark$ & $\checkmark$ & $\checkmark$ \\
\hline
\end{tabular}

are mechanical faults. Moisture, contamination, temperatures, and external vibrations also affects motor performance and considered as environmental disturbances.

Statistics of IM failures and faults due to rotor are 10\% [1] and most common cause of rotor failure is broken rotor bars, as shown in Figure 1. Causes of broken rotor bar fault of squirrel cage IM are thermal and mechanical. Thermal stresses arise due to design and construction, while mechanical stresses arise because of magnetic force and vibrations.

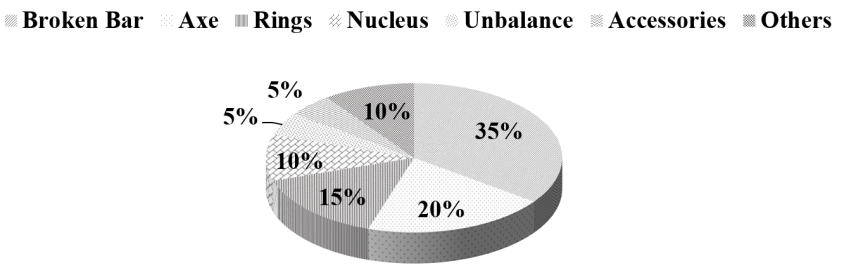

Figure 1. Problems in machine rotor

Manufacturing defects like weak joints at ends of bar, internal stresses (thermal and electromagnetic) and external constraints (frequent and overloading starts) are major causes of rotor faults. Other reasons of rotor failure are thermal overload and unbalanced mechanical stresses due to lose slots and bearing failure, magnetic stresses incurred by electromagnetic forces, dynamic loads generates thermal stresses, centrifugal forces and residual stresses are due to vibrations. These causes play pivotal part because their symptoms are detected by conventional measurement systems and affect rotating characteristics of motor [3].

\subsection{SAASMs rotor fault diagnosis}

Rotor bar is considered as most severe faults in SAASMs. Normally, these types of fault occur in heavy motors which are used in industries to drive large loads having higher inertia. Generally, these motors are very expensive and needed to maintain with no downtime. There are several causes for rotor bar breakage including centrifugal forces, electromagnetic forces, vibrations, thermal stress, electromagnetic noise, mechanical stress, fatigue parts, environmental stress, or often bearing faults. One important factor causing rotor bar is temperature gradient appearing in rotor cages and high current forces due to which current is interrupted through bars. Normally, this type of fault starts from the junction between short-circuit end rings and bars. Rotors of IM are of two types: (a) Cast, and (b) Fabricated used in squirrel-cage rotors. Cast rotors are used in high power rating machines up to 3000-kW and it's very difficult to repair after cracks or bar breakage. In case of cracked bar, current is increased by $50 \%$ of rated current in the bars. Fabricated cages are used for special application machines and for higher powers. Broken bar and cracked end-ring faults share only (5-10)\% of induction machine faults. Detection of these faults can be done by different methods, including: (a) broken rotor bar detection-current signature analysis, (b) broken-rotor-bar-induced stator current harmonics, (c) Broken-rotor-bar-detection - fault severity analysis, (d) fault severity index, and (e) broken rotor bar detection - practical considerations. For example, MCSA method is used widely to find the end- rings fault and breakage of rotor bars. Failures of rotor bars are totally analyzed from current analysis 
drawn by the machine. Rotating field theory states that when any rotor rotates at infinite inertia and at a constant speed, it generates asymmetry component of $f *(1-2 s)$ in stator current spectrum. These symmetries are mostly aligned around fundamental frequency referred as side band components given by equation below:

$$
f_{s h}=(1 \pm 2 s)
$$

where, $s$ is slip. To reduce these drawbacks, current drawn analysis of machine during transient operations is proposed by some authors also known as transient motor current signature analysis (TMCSA). In this aspect, methods based upon stator start-up current are recently introduced. Figure 2 and 3 present speed ripple effect on stator and rotor current of SAASMs due to rotor asymmetry and saturation respectively. Table 4 illustrates diagnostic techniques for different fault types and Table 5 illustrates effects of these aforementioned current components.

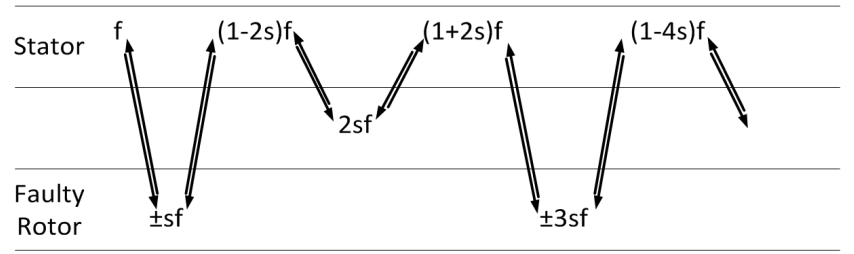

Figure 2. Effect of speed ripple on stator and rotor current of SAASMs due to rotor asymmetry

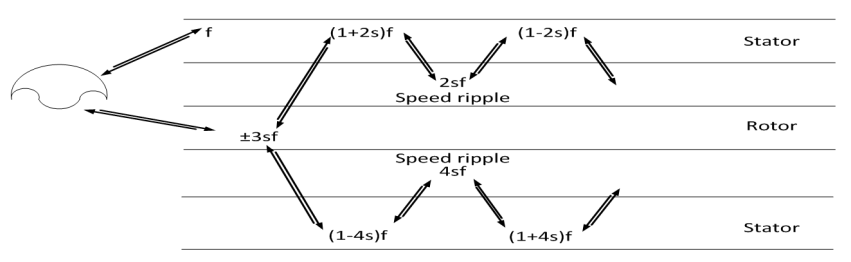

Figure 3. Effect of speed ripple on stator and rotor current of SAASMs due to saturation

Table 4. Diagnostic techniques in term of adopted signal processing techniques and input signal

\begin{tabular}{||cccc||}
\hline Type of Faults & Input Signal & Signal Processing & References \\
\hline \hline Rotor & Stator current & Frequency analysis & {$[5,12]$} \\
Rotor & Stator current & Signal injection & {$[8]$} \\
Stator & Flux & Frequency analysis & {$[15,16]$} \\
Stator & Stator current & Frequency analysis & {$[5]$} \\
\hline
\end{tabular}

Table 5. Effect of unbalanced voltages

\begin{tabular}{||ccc||}
\hline Current Component & Estimation of $n_{r}$ & References \\
\hline \hline$(1-2 s) f$ & $\frac{I_{l}}{I}=\frac{\sin \gamma}{2 P(2 \pi-\gamma)}$ & {$[17]$} \\
& $\gamma=\frac{2 \pi}{N_{r} / P} n_{r}$ & \\
$(1 \pm 2 s) f$ & $n_{r}=\frac{2 N_{r}}{10-\frac{I_{b}}{20}+P}$ & {$[18]$} \\
$(1 \pm 2 s) f$ & $\frac{I_{l}^{\prime}}{I}=\frac{I_{l}+I_{r}}{I} \simeq \frac{n_{r}}{N_{r}}$ & {$[19]$} \\
& & \\
\hline
\end{tabular}

\subsection{SAASMs stator faults}

Stator faults account for $38 \%$ of total faults of IM causing motor failure, and stator winding insulation is one of main sources of motor drive system failure. Percentage of motor drive system failures due to the 
stator winding insulation is about $26 \%$ [5]. Failure of electrical insulation system of electrical machine can be catastrophic and can result in injuries to personnel pose adverse effects on SG and also disturb economy due to shut-down processes.

\subsection{Stator insulation failure}

Stator insulation failure can occur due to different modes and patterns of failure, such as: (a) phase-to-ground failure mode, (b) turn-to-turn, (c) coil-to-coil, (d) phase-to-phase, (e) short-circuits, and (f) open-circuits. Phase to ground failure mode is most severe out of all failure modes. To find reason behind occurrence of failure mode, we need to analyze its pattern that is whether it has occurred in a single phase, is symmetrical or asymmetrical, includes or excludes grounding [5]. Figure 4 illustrates aforementioned failure modes.

\subsubsection{Turn-to-turn failure mode}

Turn-to-turn failure mode is one in which two or more turns of coil become short-circuited. Short-circuit compels current in turns to become much higher than normal operating current. Such a high current can cause temperature of stator winding to increase to levels where it can cause severe damage to insulation of winding. Majority of insulation failures starts with turn-to turn failure mode, and result in more serious insulation problems.

\subsubsection{Coil-to-coil failure mode}

In coil-to coil failure mode, two or more coils from the same phase get short-circuited, and this can be a result of turn-to-turn fault. Coils are made of several materials such as copper, plastic, steel, brass and aluminum and each having different thermal expansion coefficient. As coil cools and heats in normal operation, their thermal expansion coefficient work against each other and effectively try to tear the coil apart. Coil failure may also occur as a result of shorted winding in either primary or secondary coil. Mechanical damage should also be other failure mode to coil itself. Coils with obvious mechanical damage like cracked and broken connectors should be replaced. Except thermal cycling and damage, it may cause, there is no wear-out factor in the coil itself. Boots and wires in coil do age that result in reduced insulation with passage of time. The majority of factors related to premature induction coils failures are: (a) Operation related factors, (b) Coil material related factors, (c) design, fabrication and maintenance related factors, and (d) Accessories and tooling. Operation related factors are: (a) localized coil areas with high current density concentration, (b) inappropriate frequency, (c) improper cooling and coil overheating, (d) quenching media, (e) coil arcing, and (f) thermal expansion. Coil material related factors are: (a) copper grade selection, (b) stress fatigue cracking, (c) copper work hardening, and (d) copper erosion and pitting. Improper design, fabrication and maintenance can also cause coil-to-coil failures. Taking into consideration the aforementioned factors properly can lessen chances of coil-to-coil failures. Last group pointing out the factors related to tooling and accessories are: (a) magnetic flux concentrators, (b) quenching devices, (c) coil self-relocation, and (d) improper bus tightness.

\subsubsection{Phase-to-phase failure mode}

In phase-to-phase failure, two or more phases become short-circuited. These failure modes can subsequently lead to phase-to-ground failure due to which machine is severely damaged. Probability of occurrence of an open-circuit fault is very small. Similar to short circuit, an open-circuit initiates asymmetry in machine, thus causing machine to malfunction.

Apart from investigating the failure mode and pattern of the machine, other factors such as the cleanliness of the machine, existence of foreign particles, amount of moisture, and the rotor condition should be taken into account. Furthermore, the operating condition under which the motor failed should be compared to the normal operating condition of the motor. The schedule of machine maintenance should also be considered. The various factors that can cause the winding insulation to deteriorate include thermal, mechanical, and electrical stresses. 


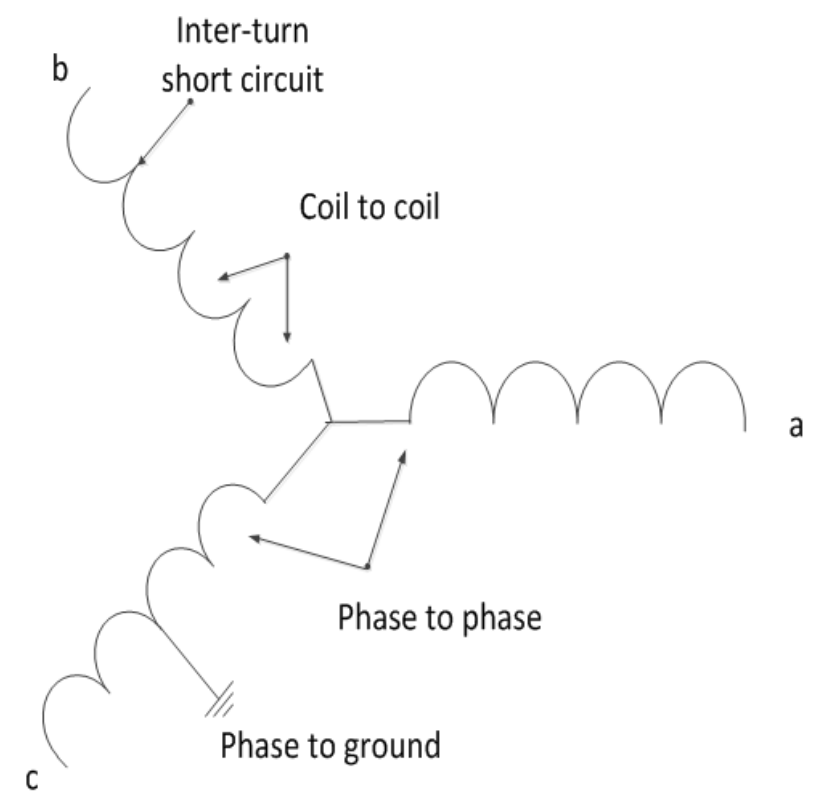

Figure 4. Failure modes of stator winding

\subsection{Thermal stresses}

Winding insulation has to undergo thermal aging referred as thermal stresses. Rise in temperature aggravates thermal aging process of winding insulation and causes it to deteriorate even further, thus reducing its lifetime. It is observed that an increase of $10^{\circ}$ in the temperature causes $50 \%$ decrease in the insulation's life [4].

In order to mitigate the effects of thermal aging, either operate motor at lower temperatures or use higher insulation class. Thermal overloading is another class of thermal stress posing detrimental effect on winding insulation. Variations in voltage, obstructions in ventilation, and imbalances in phase voltages, overloading, cycling and surrounding temperature are main causes of thermal overloading. During start-up, current is usually 3 to 5 times larger than normal operating current due to which there is rise in winding temperature. Therefore, it should be ensured that motor is not repeatedly started. An increase in load can also be a contributing factor in problem of thermal stress. Hence, load on motor that will drive must be kept in mind while designing insulation system and it must have much greater rating than normal working temperature insulation system. It is observed that if there is a voltage imbalance of $3.5 \%$ per phase, it can cause a $25 \%$ increase in temperature of phase with highest current.

To overcome effects of thermal overloading, proper ventilation of motor should be ensured so that heat produced can be dissipated without causing winding temperature to increase. If this is not possible then a better insulation with a higher rating should be chosen for stator winding.

\subsection{Mechanical stresses}

Mechanical stress causes damage to insulation when rotor strikes stator, or due to coil movement. Force exerted on winding coils is directly proportional to square of current in motor and attains its maximum value at motor start-up. Due to this force, coils begin to vibrate and move, causing serious damage to conductor and insulation of coil. Misalignment between rotor and stator, bearing faults and shaft deflection can cause rotor to strike stator. This occurs mostly during start-up, but it can also happen when motor is operating at maximum speed.

\subsection{Electrical stresses}

Electrical Stresses in motor occur due to various factors such as: (a) issues with dielectric material, (b) transient voltages, (c) effects of corona, and (d) tracking. Voltage stresses along with material used for dielectric in turn-turn, phase-phase, and phase-ground insulation greatly affect lifetime of winding insulation. Therefore, to achieve required insulation lifetime and smooth operation of motor, material for dielectric has to 
be chosen carefully. Effects of tracking occur only above voltage level of $600 \mathrm{~V}$. Transient voltages can either cause damage to winding or even complete turn-turn or turn-ground failures. Transient voltages occur due to various faults such as: (a) line-line, (b) line-ground or multi-phase line-ground faults, (c) failure of power system insulation, (d) repetitive striking, and (e) rapid bus transfer. These transients can also occur when lightning strikes, circuit breakers open and close or when switching capacitors are employed for power factor improvement. Permanent voltage transients are produced when variable frequency drives are operating, especially during starting and stopping of drives [4].

\section{Fault detection schemes in SAASMs}

\subsection{Fault detection schemes in IM}

Predictive methods are implemented in induction machines to overcome the accidental breakout. Techniques which are frequently used for the fault diagnosis in machines are (a) Speed oscillations (b) circuit analysis (c) partial discharge and (d) vibration analysis. Due to high requirement for fault diagnosis in heavy industries Electrical signature analysis (ESA) caught huge attraction of researchers to this matter. Motor current signature analysis (MCSA), vibration analysis, Instantaneous power signature analysis (IPSA) and Extended Park's vector approach (EPVA) techniques are also frequently employed by the different consultants or dedicated groups to gain better results for fault tracing in industries to enhance the efficiency of IMs. Any kind of fault in IMs can disturb its symmetry which results in to change the flux interaction between rotor and stator. Furthermore, this flux changing phenomena results in change of voltages, machine vibration, electromagnetic torque, and stator currents.

\subsection{Fault detection scheme in SM}

Different fault detection schemes are presented in literature for smooth operation of synchronous machines (SMs). These schemes provide helps regarding: (a) Ground faults detection, (b) Eccentricity faults detection, (c) Inter-turn faults detection, and (d) Phase-fault detection.

\subsubsection{Ground fault detection}

Ground-fault-detection technique for synchronous machines is presented in [16]. This technique is appropriate for synchronous machines with static excitation system and excitation field winding fed by rectifiers via excitation transformer. The said technique contributes in a way that it can detect both ac and dc side ground faults in excitation without having requirement of conventional power injection sources.

\subsubsection{Eccentricity fault detection}

In $[19,20]$, standard short circuit test is proposed for the salient pole synchronous machine to monitor the static eccentricity (SE) and dynamic eccentricity faults. The above-mentioned method possess the advantage that it based on offline current signature analysis (OFCSA) in which machine disassembly does not requires. In this technique, $\mathrm{SE}$ fault diagnosis is gained by residual estimation method for monitoring where DE is observed by manipulation of SE.

\subsubsection{Inter-turn fault detection}

Faulty machine can shut-down overall system or operation in an industry, health monitoring of machine is attracting a lot of attention recently. Due to robustness and advantage in power density in wind power generation, Permanent magnet synchronous machine (PMSM) is extensively employed due to its high robustness level and better efficiency. On-line machine monitoring fault is quite good due to fact that it is uneasy to dismantle machine once installed and cause the machine to displace. Proposed method is on-line stator winding inter-turn fault detection using electromagnetic field signature and the proposed method assists in operating movable electrical machine. 


\subsubsection{Phase Fault Detection}

A new strategy for phase fault detection of five phase permanent magnet synchronous reluctance motor (PMa-SynRM) is discussed in [20]. This fault detection technique is developed using novel decomposition process of sequential components of a five-phase electrical machine. An advance developed technique symmetrical component analysis (SCA) is implemented to trace out the phase faults in five phase or poly phase machines.

\section{Conclusions and Future Work}

Our work discussed in detail the several faults of synchronous and asynchronous machines. Induction machine is one of the most important machines playing a vital role in almost every industry due to its robustness and rigid nature. There are some common issues incurring in SAASMs as discussed in details in this paper. We investigated various rotor faults and stator faults occurring frequently in the SAASMs and affecting the efficiency of the machines resulting in a huge loss of power and uneconomical phenomenon. We also discussed several stresses that are thermal stress, mechanical stress, electrical stress and eccentricity faults and their diagnosis are also enlisted whereas fault detection techniques used in IMs and asynchronous machines are also presented. In near future, we will investigate the opening of stator phase, broken end rings, bowed shaft, broken rotor bars, offbeat rotor and bearing damage. To critically analyze the health of machine by any method, a huge information is required. For that reason, the modelling and simulation of induction motor under several conditions will be carried out in the MATLAB/SIMULINK software incorporating the stator intern-turn and broken bar conditions.

Acknowledgments: We would thankful to Prof. Dr. Muhammad Ali for providing valuable comments, suggestions and reviews for our final manuscript.

Author Contributions: All authors contributed equally to the writing of this paper. All authors read and approved the final manuscript.

Conflicts of Interest: "The authors declare no conflict of interest."

\section{References}

[1] Guzman-Sepulveda, J. R., \& Guzman-Cabrera, R. (2018). Evaluation of electromagnetic performance of emerging failures in electrical machines using computational simulation. Electrical Engineering, 100(3), 1491-1496.

[2] Hassan, O. E., Amer, M., Abdelsalam, A. K., \& Williams, B. W. (2018). Induction motor broken rotor bar fault detection techniques based on fault signature analysis-a review. IET Electric Power Applications, 12(7), 895-907.

[3] Rkhissi-Kammoun, Y., Ghommam, J., Boukhnifer, M., \& Mnif, F. (2019). Two current sensor fault detection and isolation schemes for induction motor drives using algebraic estimation approach. Mathematics and Computers in Simulation, 157, 39-62.

[4] Amirat, Y., Benbouzid, M. E. H., Wang, T., Bacha, K., \& Feld, G. (2018). EEMD-based notch filter for induction machine bearing faults detection. Applied Acoustics, 133, 202-209.

[5] Mohammed, A., Melecio, J. I., \& Djurovic, S. (2018). Stator Winding Fault Thermal Signature Monitoring and Analysis by in situ FBG sensors. IEEE Transactions on Industrial Electronics. Advance online publication https:/ / doi.org/10.1109/TIE.2018.2883260.

[6] Hannan, M. A., Ali, J. A., Mohamed, A., \& Hussain, A. (2018). Optimization techniques to enhance the performance of induction motor drives: A review. Renewable and Sustainable Energy Reviews, 81, 1611-1626.

[7] Vijayakumar, R., Mohandass, M. P., \& Angeline, S. S. (2014). An overview on performance improvement of an induction motors (im)-A review. Int. J. Innov. Res. Electr. Electron. Instrum. Control Eng, 2, 2019-2026.

[8] Zhao, Z., See, K. Y., Chua, E. K., Narayanan, A. S., Weerasinghe, A., Yang, Z., \& Tan, K. (2018, December). Online Insulation Fault Detection of Stator Winding of Induction Motor based on a Non-Intrusive Impedance Extraction Technique. In 2018 International Conference on Intelligent Rail Transportation (ICIRT) (pp. 1-5). IEEE.

[9] da Costa, C. F., Rocha, M. A., de Souza, W. G., Justino, P. T., \& Andreoli, A. L. (2018, November). Implementation of an Induction Motor Mathematical Model for Stator Winding Fault Analysis. In 2018 13th IEEE International Conference on Industry Applications (INDUSCON) (pp. 738-743). IEEE.

[10] Choudhary, A., Goyal, D., Shimi, S. L., \& Akula, A. (2018). Condition Monitoring and Fault Diagnosis of Induction Motors: A Review. Archives of Computational Methods in Engineering, 1-18.

[11] Salameh, J. P., Cauet, S., Etien, E., Sakout, A., \& Rambault, L. (2018). Gearbox condition monitoring in wind turbines: A review. Mechanical Systems and Signal Processing, 111, 251-264. 
[12] Martinez-Morales, J. D., Palacios-Hernández, E. R., \& Campos-Delgado, D. U. (2018). Multiple-fault diagnosis in induction motors through support vector machine classification at variable operating conditions. Electrical Engineering, 100(1), 59-73.

[13] Choudhary, A., Goyal, D., Shimi, S. L., \& Akula, A. (2018). Condition Monitoring and Fault Diagnosis of Induction Motors: A Review. Archives of Computational Methods in Engineering, 1-18.

[14] Hassan, O. E., Amer, M., Abdelsalam, A. K., \& Williams, B. W. (2018). Induction motor broken rotor bar fault detection techniques based on fault signature analysisâĂŞa review. IET Electric Power Applications, 12(7), 895-907.

[15] Choi, S., Haque, M. S., Tarek, M. T. B., Mulpuri, V., Duan, Y., Das, S., ... \& Toliyat, H. A. (2018). Fault Diagnosis Techniques for Permanent Magnet AC Machine and Drives-A Review of Current State of the Art. IEEE Transactions on Transportation Electrification, 4(2), 444-463.

[16] Bekhradian, R., Davarpanah, M., \& Sanaye-Pasand, M. (2018). Novel Approach for Secure Islanding Detection in Synchronous Generator Based Microgrids. IEEE Transactions on Power Delivery, 34(2), 457-466.

[17] Garcia, M., Panagiotou, P., Antonino-Daviu, J. A., \& Gyftakis, K. N. (2018). Efficiency assessment of induction motors operating under different faulty conditions. IEEE Transactions on Industrial Electronics. Advance online publication https://doi.org/10.1109/TIE.2018.2885719

[18] Mlot, A., Malloy, A. C., Korkosz, M., \& Lamperth, M. (2018). Electromagnetic Review of Rotor/Stator Misalignment in Permanent Magnet Axial Flux Motor. In Analysis and Simulation of Electrical and Computer Systems (pp. 53-69). Springer, Cham.

[19] Gyftakis, K. N., Platero, C. A., \& Bernal, S. (2018, September). Off-Line Detection of Static Eccentricity in Salient-Pole Synchronous Machines. In 2018 XIII International Conference on Electrical Machines (ICEM) (pp. 1919-1924). IEEE.

[20] Arafat, A. K. M. (2018). Analysis and control of five phased permanent magnet assisted synchronous reluctance motor drive under faults (Doctoral dissertation). OhioLINK. (akron1524168102423576) 